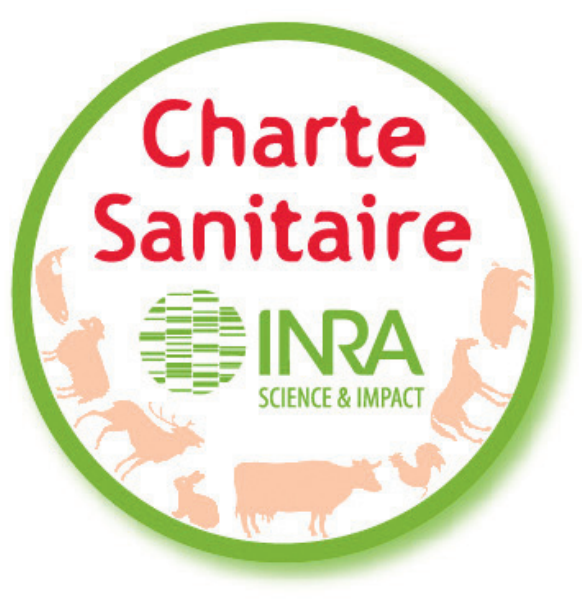

\title{
Le projet « charte sanitaire » : une politique sanitaire explicite pour les élevages expérimentaux de l'Inra
}

\author{
M. MARTIGNON 1 \\ ${ }^{1}$ INRA, Département Santé Animale, Centre Val de Loire, \\ F-37380 Nouzilly, France \\ courriel : Melanie.Martignon@tours.inra.fr
}

\begin{abstract}
Une démarche de formalisation et d'harmonisation de la gestion sanitaire dans les élevages expérimentaux de l'Inra est en cours. Elle a été initiée en $\mathbf{2 0 0 9}$ afin de fournir un cadre et un référentiel qui favorisent la coordination des actions dans le secteur de la santé animale à l'échelle de l'institut à court, moyen et long terme. Appliquée aux différentes espèces présentes à l'Inra, elle favorise aussi le partage d'expériences et les échanges d'informations en interne et avec les partenaires.
\end{abstract}

Le projet « charte sanitaire » vise à maîtriser la santé des animaux et les éventuelles conséquences des activités expérimentales et scientifiques pour la santé publique vétérinaire et l'environnement. Il s'articule autour de deux axes fondamentaux :i) le respect des réglementations auxquelles sont soumis les élevages expérimentaux de l'institut et $i i$ ) une sensibilisation des agents aux risques sanitaires liés à l'expérimentation en élevage.

\section{1 / Périmètre et enJeuX du Projet « CHaRte SANitaire »}

La mise en place de la charte sanitaire implique tous les intervenants (animaliers, personnels des unités et scientifiques utilisateurs, vétérinaires, inséminateurs, pareurs, maréchaux-ferrants...) dans les dispositifs expérimentaux de l'Inra. Toutes les espèces (oiseaux, lapins, bovins, ovins, caprins, équins, porcins et poissons) sont concernées par la démarche, sauf les animaux modèles (rongeurs et poissons modèles) dont la gestion sanitaire est déjà très encadrée.

Ces cheptels ont une haute valeur scientifique, dans la mesure où ils conditionnent le devenir de programmes de recherche de l'Inra, pour la réalisation desquels certains animaux ont été sélectionnés depuis plusieurs générations sur un ou plusieurs caractères d'intérêt. L'originalité de ce projet est de créer une politique de gestion sanitaire générique, tout en tenant compte des spécificités de chaque élevage représenté. Ces spécificités s'expriment par exemple en termes d'espèce hébergée, de milieu de vie (aquatique, terrestre), de système d'élevage (agriculture biologique, élevage hors-sol, élevage extensif...), de proximité de la faune sauvage, de fréquence des manipulations, de thématiques de recherche traitées et/ou de contraintes géographiques.

Il s'agit, dans un premier temps, d'informer et de sensibiliser les agents intervenant dans les élevages expérimentaux sur la réglementation et son évolution et sur les bonnes pratiques garantissant leur sécurité, la pérennité des cheptels et l'intégrité de l'environnement. Dans un second temps, la connaissance du statut sanitaire des élevages doit renforcer la fiabilité des résultats d'expérimentations et encadrer les mouvements d'animaux entre sites expérimentaux. Enfin, formaliser les pratiques éthiques au sein des élevages expérimentaux garantit le respect du bien-être des animaux dont nous avons la responsabilité. De plus, la formalisation des pratiques et la rédaction de procédures facilitent l'accueil de visiteurs et l'intégration de nouveaux arrivants dans les dispositifs expérimentaux. Les informations sont ainsi aisées à transmettre et elles leur garantissent d'évoluer dans ce nouvel environnement en respectant les règles.

Encadré 1. Membres du collectif « Charte sanitaire » (à la date du 01/12/2013).

Comité de pilotage : Didier Azam, Xavier Berthelot, Jean-Luc Cadoré, Séverine Deretz, Stéphane Ingrand, Guy-Pierre Martineau, Thierry Pineau, Pierre Sarradin, Jean-Marie Trommenschlager.

\section{Groupes de travail :}

Bovins : Sébastien Assié / Damien Capo, Delphine Caray, Franck Chargelègue, Jérôme Fleury, Emilie Fombaron, Yves Gallard, Jacques Lassalas, David Maupetit, Ludovic Métivier, Christophe Rossignol.

Équins : Jean-Luc Cadoré / Fabrice Reigner.

Lapins : François Tudela / Gérard Auvinet, Virginie Héliès, Nicolas Stadler.

Petits ruminants : Xavier Berthelot, Bruno Polack / Mathias Aletru, Claude Barbier, Karine Boissard, Yves Bourdillon, Damien Capo, Delphine Caray, Hervé Cassard, Nicolas Cébé, Didier Crochet, Didier Dubreuil, Alexandra Eymard, Thierry Fassier, Jacques Lassalas, Jean-Marie Menras.

Poissons : Christian Michel / Frédéric Borel, Laurent Espinat, Antoine Gallard, Stéphane Glise, Lionel Goardon, Dimitri Rigaudeau, Frédéric Vallée.

Porcins : Guy-Pierre Martineau / Bruno Bocage, Hervé Demay, Stéphane Ferchaud, Philippe Gerbe, Nelly Muller, Jonathan Savoie. Volailles : Jean-Luc Guérin / Jean-Marie Alletru, Marie-Dominique Bernadet, David Gourichon, Laurence Mérat, Jean-Marc Meslier. 
A l'instar des démarches qualité, la charte sanitaire s'appuie sur un réseau d'intervenants structuré, aisément mobilisable et ancré dans le fonctionnement de l'Institut. Elle vise à élaborer et généraliser un référentiel au sein des élevages expérimentaux et s'accompagne d'actions périphériques complémentaires. Le réseau d'acteurs (cf. encadré 1) est constitué d'un comité de pilotage qui fixe les orientations du projet, d'une coordinatrice de projet et de groupes de travail par espèce. Ces groupes de travail réunissent les correspondants sanitaires de chaque élevage, relais du projet pour leur élevage et inversement; ils sont animés par des vétérinaires spécialistes de l'espèce considérée.

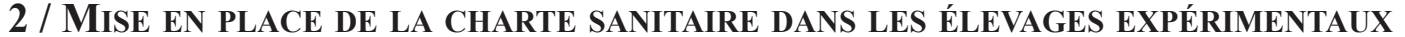

\section{1 / Une rédaction collaborative du référentiel}

L'harmonisation des pratiques sanitaires dans les élevages expérimentaux de l'Inra s'appuie sur un référentiel intitulé « la charte sanitaire Inra ». Celui-ci est constitué d'un tronc commun à toutes les espèces, qui compte 6 articles (cf. encadré 2). Il rappelle les fondements et les grands principes de la démarche. Ce tronc commun est complété par des annexes déclinées par espèce, chacune étant divisée en trois parties : hygiène et/ou bientraitance, suivi sanitaire des cheptels et maîtrise des risques sanitaires. La charte sanitaire Inra permet de synthétiser en un seul document les engagements réglementaires pris dans les dispositifs expérimentaux, ainsi que les bonnes pratiques d'élevage qui les concernent et de proposer des moyens simples pour leur mise en œuvre. Par exemple, des actions d'accueil et de prise en charge des visiteurs et des nouveaux arrivants sont proposées : enregistrement, fourniture d'équipements de protection et affichettes d'information sur les consignes à appliquer.

Encadré 2. La « charte sanitaire Inra »-Engagements communs.

\section{Article 0 : Réglementation sanitaire}

Afin de veiller au respect de la réglementation sanitaire dans les dispositifs expérimentaux, les obligations relatives $i)$ aux risques majeurs impliquant des mesures de police sanitaire, ii) au suivi des cheptels et à la traçabilité des informations sanitaires, iii) à la gestion des effluents, déchets infectieux et toxiques, iv) aux modalités de prescription, de délivrance et d'utilisation du médicament vétérinaire et $v$ ) aux conditions et pratiques d'élevage, indissociables d'une dimension éthique (relation étroite entre bien-être et santé de l'animal), doivent être régulièrement identifiées (veille règlementaire) et précisées.

\section{Article 1: Risques sanitaires spécifiques aux activités d'élevage et de recherche de l'Inra}

Afin de garantir la sécurité des cheptels, des personnels et de l'environnement, ainsi que la qualité des expérimentations conduites, les risques sanitaires particuliers liés aux exigences des activités d'élevage et de recherche de l'Institut doivent être régulièrement définis. II s'agit : i) des risques de zoonose, ii) des risques sanitaires ayant un impact sur les activités expérimentales et d'élevage et iii) des risques de contamination environnementale. L'identification et l'évaluation de ces risques doivent considérer : i) les mouvements d'animaux, gamètes et produits d'origine animale (traçabilité), ii) les mouvements des différents intervenants dans l'élevage (animaliers, scientifiques, vétérinaire, laiterie...), iii) la gestion des déchets et effluents iv) la conduite d'élevage, l'hygiène et la conception des infrastructures et $v$ ) les protocoles expérimentaux mis en place.

\section{Article 2: Gestion et conduite sanitaire}

Afin de gérer les risques sanitaires identifiés (élimination ou prévention), des pratiques adaptées et formalisées doivent être mises en œuvre et intégrées dans la démarche qualité et prévention, en place au sein des unités. Ces pratiques doivent faire l'objet d'une constante évaluation, en vue de leur mise à jour et amélioration. Dans ce cadre, il s'agit : i) de définir un cahier des charges de suivi sanitaire et de gestion des maladies animales, en collaboration avec le vétérinaire traitant, les scientifiques et chercheurs concernés (calendrier de visites, procédures de diagnostic et définition du statut sanitaire, utilisation des médicaments, gestion de crise sanitaire), ii) de définir un schéma d'introduction et de distribution des animaux, des gamètes et produits animaux, iii) de définir un schéma d'élimination et d'évacuation des effluents et déchets infectieux et toxiques et iv) de développer un dispositif de traçabilité des informations sanitaires adapté aux espèces et structures de l'unité.

\section{Article 3: Transparence des statuts sanitaires au sein de l'Inra}

Dans un but de transparence, indispensable notamment aux échanges d'animaux entre unités, les informations sanitaires relatives aux dispositifs expérimentaux doivent être communiquées au sein de l'Inra. Pour cela, un bilan sanitaire régulier est effectué auprès des instances légitimes.

\section{Article 4 : Implication des acteurs}

Pour une gestion sanitaire intégrée et harmonisée, les unités et installations expérimentales doivent travailler en collaboration avec les différents acteurs partenaires, internes et externes à l'Inra (scientifiques utilisateurs des cheptels, missions prévention et formation permanente, délégation qualité, autres dispositifs expérimentaux, vétérinaire(s), autorités sanitaires, Groupements de Défense Sanitaire...). Pour cela, le (les) correspondant(s) sanitaire(s), identifié(s) dans chaque dispositif, interagit(ssent) avec les acteurs partenaires impliqués en fonction des mesures engagées.

L'ensemble des intervenants dans les cheptels doit par ailleurs s'attacher à respecter les dispositions mises en œuvre dans le cadre de la charte.

Enfin, afin de pouvoir s'appuyer sur des expertises spécifiques, des spécialistes sont identifiés, par espèce, voire par agent pathogène, au sein de l'Inra, mais aussi à l'extérieur de l'institut. Ces experts interviennent lors d'audits conseils, à l'occasion de formations et, d'un point de vue plus général, sont consultés dans le cadre des réflexions sur le contenu de la charte et sa mise en œuvre.

\section{Article 5 : Développement de compétences}

La maîtrise du statut sanitaire des installations expérimentales passe par une sensibilisation de l'ensemble des personnels concernés, par une diffusion des informations sanitaires et par l'acquisition de compétences adaptées. Pour cela, des formations spécifiques seront construites et des partages d'expérience entre les différents partenaires, de l'Inra ou extérieurs, seront organisés, en cohérence avec les plans de formation des unités. 
Le mode de rédaction du référentiel est représentatif de la philosophie de la démarche qui se veut participative et au service du collectif. En effet, chaque annexe a été rédigée par un trinôme incluant un vétérinaire spécialiste de l'espèce considérée, un correspondant sanitaire et la coordinatrice du projet. Les documents ainsi élaborés ont été soumis à l'avis de l'ensemble des représentants des élevages impliqués dans la démarche. Ils ont ainsi pu s'approprier la démarche et l'adapter aux contraintes propres à leur situation. Ainsi, ce référentiel représente une base commune et consensuelle d'engagements formalisant les pratiques d'élevage au sein de l'institut.

\section{2 / Une mise en application appuyée sur le réseau « charte sanitaire »}

La mise en place du référentiel dans chaque élevage repose sur les correspondants sanitaires qui s'appuient sur $i$ ) le soutien indispensable des directeurs et responsables des élevages expérimentaux concernés, ii) la motivation des équipes et iii) la sensibilisation de tous les intervenants en élevage. Les correspondants sanitaires évaluent régulièrement l'avancement de la démarche dans leur élevage et proposent des plans d'action qu'ils soumettent à l'avis de leur direction. Ces plans d'action hiérarchisent les points à améliorer et proposent des actions et des échéances pour chacun. Ces auto-évaluations régulières permettent d'améliorer les pratiques en continu et selon un rythme tenant compte de leurs contraintes, exigences propres et degrés d'avancement. Cette adaptabilité de la démarche est à souligner, car elle permet de faciliter sa mise en œuvre et son appropriation dans des contextes variés.

\section{3 / ACTIONS COMPLÉMENTAIRES}

Dans le cadre de ce projet, des informations de veille sanitaire sont diffusées régulièrement aux membres du réseau. Ainsi, les personnes concernées sont tenues informées de l'évolution de la réglementation et des évènements sanitaires en cours (par exemple, la maladie de Schmallenberg chez les ruminants en 2011-2012). Les textes de référence mis à disposition dans le référentiel sont actualisés annuellement.

Des guides techniques sont également rédigés, sur certains thèmes communs à tous les élevages, pour appuyer la mise en place du référentiel. Ainsi, le guide technique détaillant la gestion de la pharmacie vétérinaire dans les cheptels de l'Inra a été diffusé fin 2010. Sur ce point, en effet, les élevages de l'Inra sont concernés par deux domaines réglementaires (élevage et expérimentation animale) et il était important de clarifier les conditions justifiant le recours à l'un ou l'autre. Un autre guide technique sur la sécurité biologique est en réflexion, en collaboration avec certains responsables « santé et sécurité » au travail de l'institut.

Un outil informatique est en cours de développement. Ce module appelé CaSaME - Carnet Sanitaire Multi-Espèces - est dédié à la gestion sanitaire dans les élevages expérimentaux et compatible avec les logiciels de gestion d'élevage. Il répond à trois objectifs principaux : $i$ ) appuyer les élevages expérimentaux de l'institut dans le respect de la règlementation, $i i)$ les aider à la mise en place du référentiel « charte sanitaire » et iii) faciliter la gestion des opérations quotidiennes de soins aux animaux ou pour répondre à des besoins expérimentaux spécifiques. Par exemple, le logiciel facilitera la gestion du stock de pharmacie selon les législations en vigueur, en permettant la saisie d'un évènement sanitaire auquel pourra être associé un traitement choisi directement dans la pharmacie de l'élevage.

Des actions d'animation sont organisées pour favoriser les échanges entre les membres du réseau répartis sur le territoire national. Elles permettent aux correspondants sanitaires de mutualiser les documents et les méthodes utilisées ainsi que de partager leurs expériences. Par ailleurs, des journées thématiques sont programmées chaque année. Les informations transmises lors de ces journées consolident les connaissances des correspondants sanitaires sur un thème de leur choix.

Enfin, l'avis des experts du réseau est sollicité dès les premières phases des projets de construction de bâtiments d'élevage ou autres installations expérimentales. Le rappel des contraintes réglementaires et des principes de protection de la santé des animaux peut alors être pris en compte en amont des travaux. Les avis donnés concernent en particulier les conditions d'ambiance (ventilation et température), la circulation des animaux dans les bâtiments et la cohérence de l'organisation des salles d'élevage selon les stades physiologiques et/ou les statuts sanitaires (par exemple, en évitant d'héberger des jeunes à proximité de l'infirmerie).

\section{4 / Conclusions}

Le projet « charte sanitaire » vise à formaliser les bonnes pratiques de l'Institut en matière de gestion sanitaire des élevages et de bientraitance des animaux. Elle garantit en outre la fiabilité des résultats scientifiques et permet la transparence de l'Institut concernant ses élevages.

Malgré la complexité des enjeux sous-tendant le projet et la diversité des situations, l'écoute des besoins des acteurs et leur prise en compte dès l'élaboration du projet, puis en continu lors de son déploiement, renforcent la cohésion autour de cette démarche générique et garantissent sa faisabilité.

Cette démarche a d'abord été élaborée pour une application dans les élevages de l'Institut, mais elle intègre toutes les composantes de la vie d'un élevage expérimental, qu'il s'agisse de l'intervention de professionnels spécialisés extérieurs à l'Institut ou l'accueil de visiteurs ou de stagiaires. Conçue à la base pour s'adapter à des contextes diversifiés (espèces, environnement...), la démarche est facilement généralisable à tout élevage ayant des problématiques de gestion sanitaire proches. 